\title{
Preoperative surgical planning for an internal carotid artery invading brain tumor
}

\author{
Hendrikus Masang Ban Bolly,2, \\ Bilzardy Ferry Zulkifli', \\ Achmad Adam' \\ 'Department of Neurosurgery, Faculty of \\ Medicine, Universitas Padjadjaran-Dr. Hasan \\ Sadikin Hospital, Bandung, Indonesia \\ ${ }^{2}$ Department of Biochemistry Faculty of \\ Medicine, Cenderawasih University, Jayapura, \\ Indonesia
}

\begin{abstract}
Brain tumor such as a meningioma can invade the adjacent internal carotid artery (ICA). Surgical approach to resect the tumor has to consider the level of ICA and how the depth of tumor cells invade into the artery layer. Knowledge of the basic mechanism about how the tumor cells invade the lumen and layer of the vessels is essential in perioperative preparation. Here we reported our unpredictable case findings of transient intraluminal obstruction of the arterial wall by tumor tissue in angiography examination as well as preoperative planning to resect the tumor and predictors of the patient's outcome.
\end{abstract}

Keywords: Angiography, Intraluminal, Meningioma

\section{Introduction}

Knowledge of the possible invasion of the tumor to ICA is essential for the skull base surgeon to accurately plan the surgical approach since the meningioma may surround the ICA and invade its wall outside and inside. ${ }^{1}$ The neurosurgeon has to find a way to access the tumor safely without disrupting the arterial wall. Because of the importance of the anatomical landmark and possibility of an invasion of ICA wall by the tumor, the evaluation between the interface of tumor and ICA in cases of arterial luminal stenosis of ICA has to make clear in perioperative planning. The tumors can intercalate in the adventitial layer of the carotid artery with subjacent stenosis of the arterial lumen. Edema can found within the tunica media, and elastic lamina can be disrupted by the tumor cell then focally extend into the muscular media. ${ }^{1}$ Hendrikus Masang Ban Bolly Department of Neurosurgery, Faculty of Medicine Universitas Padjadjaran Dr. Hasan Sadikin Hospital, Bandung, Jawa Barat, Indonesia hendrikusbolly@gmail.com
In this report, the authors describe the gross anatomy of the internal carotid artery of clinoidal segments based on the digital subtraction angiography (DSA) findings, the basic mechanism of how the tumor invades to the arterial wall also reviewed in the context of perioperative surgical planning to tumor resection in clinoidal area. Understanding the anatomy and basic molecular characteristic of tumor metastasis was very important for successful surgical planning. 


\section{Case Report}

A 29-year-old woman was referred to perform a DSA examination. She had a complaint of blurred vision of the left eye about a month before the procedure. History of chronic headache had complained since two years before, and there was no other clinical complaint until she came to the hospital for the blurry left eye. There were no other symptoms of cranial nerve palsy. The patient had no history of seizures, fever, or unresponsiveness. Axial CT scan without the Gadolinium contrast showed a hyperdense mass in the clinoidal area, circular and had a clear border to brain parenchymal tissue. We underwent a DSA examination to exclude the differential diagnosis of a giant aneurysm and to study the anatomical structure of ICA closed to the clinoidal mass.
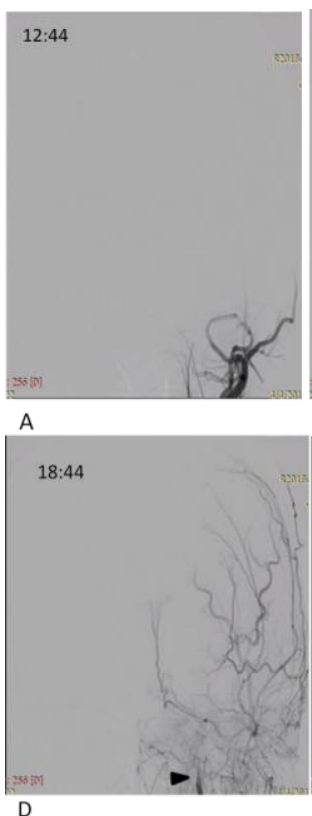

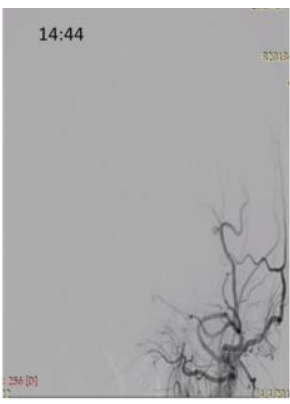

B

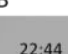

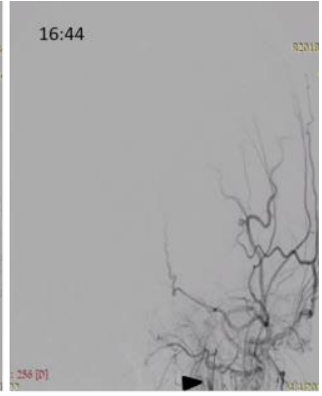

c

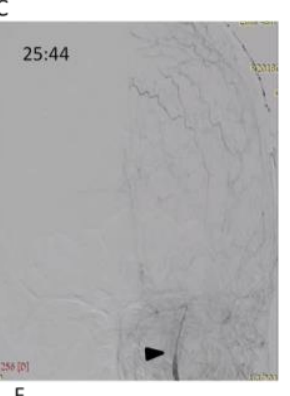

Figure 1. Serial left common carotid injections of contrast

A and B. Initial views, there is no flow of left ICA while left ECA already filled by the contrast.

C.First flow in the left ICA. Early filling of the branches of left ECA but delayed filling of left ICA

D and E. Delayed filling of left ICA $(\rightarrow)$ while almost of left ECA filled by contrast.

F. Delayed filling of ICA of the vasculature, show there is a transient obstruction with irregular pattern of left ICA and a blockage of the flow

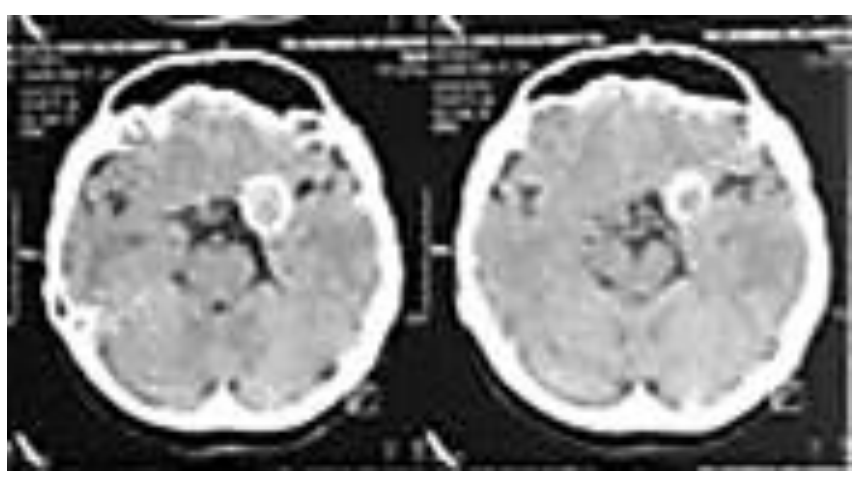

Figure 2. Axial CT scan of the patient showed a typical clinoidal meningioma

\section{Discussion}

We suspected the tumor as the clinoidal meningioma. This kind of tumor is usually originating from the anterior clinoid bone or a medial third of the sphenoid ridge. Total removal of huge clinoidal meningioma is a highly complex surgical procedure because of the tumors frequently encase large cerebral vessels of ICA and its branches. These tumors also can infiltrate the adventitia of the ICA, invade the cavernous sinus or the infratemporal fossa. The most common clinical manifestations of clinoidal meningiomas are visual disturbances and headaches. ${ }^{2}$ Some other clinical symptoms will exist related to the increase of tumor size and the extent to the parasellar area. Clinoidal meningioma is found more common in females than males. The surgical procedure to remove clinoidal meningiomas need a fully comprehensive understanding of adjacent neurovascular structure. ${ }^{2}$

There are three types related to the site of origin and adhesiveness of the tumor to the internal carotid artery and its branches. Type I arise from the subclinoidal dura at the most proximal point of intradural entry of ICA. Type II originates from the superolateral aspect of the anterior clinoid process. Type III originate from the region of the optic foramen and extent into the optic canal. ${ }^{2}$

The ICA supplies the ipsilateral cerebral hemisphere, sensory organs and eye, forehead, and nose. ${ }^{2,3}$ The recent classifications of the Bouthillier consist of 7 main segments, i.e., cervical, petrous, lacerum, cavernous, clinoid, ophthalmic, and communicating. ${ }^{3,4}$ Clinoid segment of the ICA located between the cavernous and the intradural compartment. It was located and covers by the carotid-oculomotor membrane. We could find a lateral part of ICA at the clinoid segment after removed the anterior clinoid process; otherwise, the medial part of the ICA was not covered by the clinoid bone. ${ }^{3}$ Related to preoperative evaluation of CT scan and DSA that performed in this patient, the plan of gross total resection sometimes cannot be achieved. Based on Sughrue et al., ${ }^{1}$ Simpson grade 1-3 sometimes can not be done because of optic canal invasion, vessel encasement and sellar invasion of the clinoidal tumor.

In our case, we found that the artery lining became narrow partially. The contrast substrate flowed slowly in the intravascular. Tumor cells could be found in the adventitia of the artery with stenosis of the arterial lumen. ${ }^{5}$ The tumor also could invade the elastic lamina of vascular. We could predict that the tumor had invaded the lumen of ICA. The invasion of the carotid wall in the clinoidal by a tumor (such as meningiomas) occurred as a consequence of the absence of arachnoidal plane in that area. ${ }^{5}$ Tumor invasion to the ICA structure had 
never been observed in the intradural space because of the arachnoidal part separated the tumor from the adjacent artery. 5,8

Based on the review of Shaffrey et al., ${ }^{5}$ the local factor facilitated the tumor invasion into the carotid arterial wall because of the anatomical structure of clinoid. Initial local compression needs an extended period. At first, the tumor attacks the outer-lining of the artery, and after there is no more space for its growth, tumor cells start to fill into the artery's lumen. Based on the histology appearance, tumor cells can compress and obliterate the adventitial layer of the vessels. In further, tumor cells invade the media muscle of the vessels and change the morphological of the vascular lining. In the end, we found that the degeneration changing and had a higher risk to become vascular rupture..$^{5}$

Review of Krakhmal et al., ${ }^{6}$ rules out the two patterns of cancer cell invasion, i.e. collective cell migration and individual cell migration. The migration type at the end will be determined by tissue microenvironment and fundamental molecular changes of tumor cells. ${ }^{5,6} \mathrm{We}$ suspected that the mode of meningioma invades the vessels through the collective cell migration and invasion. The process begins in chronic prolonged contact and adherent of the tumors cells to their neighbor's vessels. These processes involved some protein include cadherin, tight junction proteins, cytoskeletal protein, and signaling process. ${ }^{7,10}$ Another contributor related to the risk factors for metastases of meningioma such as high cellularity, cellular heterogeneity, high mitotic rate, nuclear pleomorphism, tumor necrosis and invasion of adjacent blood vessels. ${ }^{8,10,11}$ As we suspected, the tumor is meningioma and meningioma has three distinct classifications by WHO as benign (grade I), atypical (grade II) and anaplastic (grade III); the risk of invasion spreads depend on that grading. ${ }^{8,9}$ Some extra-cranial metastases also can arise in lungs (60\%), abdomen and liver (34\%) cervical lymph nodes (18\%), long bones (11\%), pleura 9\%), vertebrae (7\%) and mediastinum (5\%). The dissemination of tumor cells has four different ways, hematogenous, paravertebral venous plexus, lymphatics, and cerebrospinal fluid. ${ }^{8}$

Since DSA showed that our patient already had the tumor in the vessel wall and intraluminal, the risk of distant metastases increased, this reality also suggests us to plan the perioperative carefully.

\section{Pre-operative planning and Outcome}

Al-Mefty recommendation for clinoidal meningioma removal is wide frontoorbital craniotomy. ${ }^{2}$ Salunke et al., describe a technique of frontotemporo orbitozygomatic craniotomy and drilling the clinoid bone extradural. ${ }^{12}$ Using the micro drill, we have to expose the superior orbital fissure and transect the meningoorbital band to facilitate the access to anterior clinoid processus. After that, in the extradural procedure, we have to unroof the optic canal and exposed the optic nerve. At this point, we already achieve a complete clinoidectomy. We can identify an optic nerve in the medial part, the clinoidal segment of ICA in the lateral and inferiorly to the optic nerve. ${ }^{13}$ The surgeon has to be aware of the risks and complications include visual disturbances, oculomotor paresis, injury to the optic nerve or injury to the ICA itself after anterior clinoidectomy. ${ }^{3}$ We can open the dura mater in the lateral part of the optic canal and continue in a medial direction to the optic nerve directly to the falciform ligament, in the lateral direction to the distal carotid ring. ${ }^{12,13}$ In the intradural portion, we have to identify some important structures of prechiasmatic part of the optic nerve, supraclinoidal segment of ICA and the cranial nerve III lateral to the ICA. ${ }^{13}$

Preoperative identification of the vascularization that supplies the meningioma is really important. The surgeon has to de-attach the feeder artery firstly. Luminal narrowing of ICA finding like, in this case, will affect the specific planning to resect the tumor. The conceal of distal ICA, bifurcation of ICA and proximal anterior cerebral artery (A1 segment) - middle cerebral artery (M1 segment) by tumor tissue is the "dangerous triad". ${ }^{12}$ The surgeon must have a good plane of dissection among the tumor and luminal cramping artery that showed in the angiogram. This is why the identification of tumor encasement and luminal cramping of the ICA are important pre-operative matter to decide the extension of the resection. ${ }^{12}$ Some studies indicate an unfortunate result of resection if a longer artery within the tumor and include the luminal cramping exist. Luminal cramping of ICA is the sign of tumor infiltration into the ICA and make the resection riskier. ${ }^{14,15}$ The clinoidal meningioma still face a challenge due to its close location to ICA and its major branch, optic and oculomotor nerves. ${ }^{16}$ Al-Mefty divided the clinoidal meningioma into three groups. In type I tumor, it is better not to resect the tumor from the vessel because the tumor grows along the artery and peel the arachnoid surround the vessels. ${ }^{12}$ This type is the toughest because of having a higher risk of vascular injury. Useful facts from the study of Salunke $e t$ $a l$., that involved the 21 cases of anterior clinoidal meningiomas found that the plane between the ICA and tumor could be separate instead there are a complete encasement and cramping of the artery. ${ }^{12}$ The preoperative pattern of vascular encasement by tumor tissue in DSA is important but more important is intraoperative finding. Studies show that the tumor can adhere to the proximal level of clinoidal ICA and extent to the proximal A1 segment and M1 segment. Tumor tissue that 
invades the adventitia layer of the vessels has a higher risk of vessel injury and rupture. M1 rupture related to delayed hemiparesis after the operation. Another intraoperative complication that involved ICA cramping by the tumor is spasm and related to postoperative ischaemic complications. ${ }^{12}$ The study of Salunke et al., in 21 patients of clinoidal meningioma involving the ICA cramping and luminal narrowing show the results of safe maximal resection after a good plane dissection. ${ }^{12}$ They also found that segmental narrowing of the ICA in preoperative DSA sometimes not proven intraoperatively, despite the fact that the surgeon has to anticipate if vascular conceal near the area of bifurcation.

We reported the complaint of blurred vision of the left eye in our patient. Informed consent related to the morbidity of this complaint after the tumor resection is essential in the preoperative period. Visual deterioration into more bad conditions caused by ischemic injury of blood suppliers to the optic apparatus. As we mentioned before, there are some risky of vessel injury, rupture and spasms. We have to more aware and prevent all the dangerous complications by having a good knowledge of the standard and pathological aspect of anatomy and physiology of this tumor. ${ }^{2}$ Tumor recurrence and progressions are similar to other meningioma and all depends on the extension of first tumor resection. The fact of the vascular infiltration by tumor tissue will support the possibility of distant metastases spreading of the tumor. ${ }^{8}$ On the other side, a history of re-craniotomy also increases the risk of metastases.

Regarding the technique of craniotomy, a frontoorbital approach has a better angle to remove the clinoidal tumor but has a disadvantage of a higher rate of transient postoperative ptosis. ${ }^{13}$ A postoperative complication is pseudomeningocele and does not need surgical intervention. On average, it needs about five weeks to a spontaneous resolve. A frontoorbital approach for these meningioma results in $36.3 \%$ of pseudomeningocele complications. In other study show a different number, Behari et al., ${ }^{17}$ report $20 \%$ of pseudomeningocele complications using a frontotemporal approach; Nakamura et al., ${ }^{18}$ report $6.5 \%$ of these complications using a pterional and frontolateral craniotomies.

The study of Attia et al., ${ }^{13}$ report a good outcome of a GOS score of 5 in $86.3 \%$ using a frontoorbital approach. These approaches provide a good extent resection, good visual, and clinical outcomes.

\section{Conclusion}

We found that the tumor cells probably already invade the intraluminal and the wall of ICA. The neurosurgeon has to plan a better approach to resect the tumor and salvage the encase vessels and narrowing luminal. This preoperative angiogram is essential for planning and increases surgeon awareness when dissecting the tumor intraoperatively. A frontoorbital approach of craniotomy for this patient perhaps can achieve a good clinical outcome.

\section{Acknowledgment}

All authors declare there is no potential conflict of interest relevant to this article. There is no specific grant supported for this works or article.

\section{References}

Sughrue M, Kane A, Rutkowski MJ, et al. Meningiomas of the anterior clinoid process: Is it Wise to drill out the optic Canal?. Cureus. 2015;7(9): e321. DOI: 10.7759/cureus.321

2. Krisht AF. Clinoidal meningiomas. In: Franco D, Michael WM, Ossama A (Eds). Al-Mefty's Meningiomas. Second Edition. New York: Thieme, 2011, pp 228-36

3. Guclu B, Sindou M. Surgical access to the paraclinoid segment of the internal carotid artery: a technical review. Sinir Sistemi Cerrahisi Derg. 2014;4(1):1-8. DOI: 10.5222/sscd.2014.001

4. Shapiro M, Becske T, Riina HA, et al. Toward an endovascular internal carotid artery classification system. AJNR Am J Neuroradiol. 2014;35(2):230-6. DOI: 10.3174/ajnr.A3666

5. Shaffrey ME, Dolenc VV, Lanzino G, et al. Invasion of the internal carotid artery by cavernous sinus meningiomas. Surg Neurol. 1999;52(2):167-171. DOI: 10.1016/50090-3019(99)00092-0

6. Krakhmal NV, Zavyalova MV, Denisov EV, et al. Cancer invasion: patterns and mechanisms. Acta Naturae. 2015;7(2):17-28. DOI: 10.32607/20758251-2015-7-2-17-28

7. Friedl P, Locker J, Sahai E, et al. Classifying collective cancer cell invasion. Nat Cell Biol. 2012;14(8): 777-783. DOI: $10.1038 /$ ncb2548

8. Kanthan R, Senger JL. Distant metastases from meningiomas-a myth or reality? Ann Clin Pathol. 2013;1(1):1001. Available at: https://www.jscimedcentral.com/Pathology/Articles/pathology-1-1001.pdf

9. Riemenschneider MJ, Perry A, Reifenberger G. Histological classification and molecular genetics of meningiomas. Lancet Neurol. 2006;5(12):1045-54. DOI:10.1016/S1474-4422(06)70625-1

10. Jagannathan J, Oskouian RJ, Yeoh HK, et al. Molecular biology of unreresectable meningiomas: implications for new treatments and review of the literature. Skull Base. 2008;18(3):173-87. DOI: 10.1055/s-2007-1003925

11. Z Zijl F, Krupitza G, Mikulits W. Initial steps of metastasis: Cell invasion and endothelial transmigration. Mutat Res. 2011;728(1-2):23-24. DOI:10.1016/j.mrrev.2011.05.002

12. Salunke P, Singh A, Kamble R, et al. Vascular involvement in anterior clinoidal meningiomas: Biting the 'artery' that feeds. J Clineuro. 2019;184:105413. DOI: 10.1016/j.clineuro.2019.105413.

13. Attia M, Umansky F, Paldor I, et al. Giant anterior clinoidal meningiomas: surgical technique and outcomes. $J$ Neurosurg. 2012;117:654-65. DOI: 10.3171/2012.7.JNS111675

14. Goel A, Gupta S, Desai K. New grading system to predict resectability of anterior clinoid meningiomas. Neurol Med Chir (Tokyo). 2000;40:610-16. DOI:10.2176/nmc.40.610

15. Ishikawa M, Nishi S, Aoki T, et al. Predictability of internal carotid artery (ICA) dissectability in cases showing ICA involvement in parasellar meningioma. J Clin Neurosci. 2001;8 (Suppl 1):22-25. DOI:10.1054/jocn.2001.0872

16. Kim J, Jang W, Jung T, et al. Predictive factors for surgical outcome in the anterior clinoidal meningiomas. Medicine. 2017;96:15(e6594). DOI: 10.1097/MD.00000000000006594

17. Behari S, Giri PJ, Shukla D, et al. Surgical strategies for giant medial sphenoid wing meningiomas: a new scoring system for predicting the extent of resection. Acta Neurochir (Wien). 2008;150:865-77. DOI: 10.1007/s00701-00800066

18. Nakamura M, Roser F, Jacobs S, et al. Medial sphenoid wing meningiomas; clinical outcome and recurrence rate Neurosurgery. 2006;58:626-39.DOI:10.227/01.NEU.0000197104.78684.5D

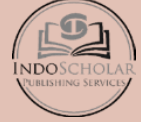

Articles published online under this model are officially published and can be cited and quoted using the DOI as the reference source. Neurologico Spinale Medico Chirurgico has a policy that changes will not be made after publication of an article without following accepted procedures for making corrections to the scientific record. The entire contents of the Neurologico Spinale Medico Chirurgico are licensed under the Creative Commons Attribution 4.0 International License. You are free to share - copy \& redistribute the material in any medium or format, adapt - remix, transform, and build upon the material for any purpose, even commercially. Under the following terms, you must give appropriate credit, provide link to the license, and indicate if changes were made. You may do so in any reasonable manner, but not in any way that suggests the licensor endorses you or your use.
} 